\title{
Finite volume computation for the non-stationary probability density function of an impulsively controlled 1-D diffusion process
}

\author{
Yuta Yaegashi $^{1,2}$, Hidekazu Yoshioka ${ }^{3,4^{*}}$, Motoh Tsujimura ${ }^{5}$, Masayuki Fujihara ${ }^{1}$ \\ ${ }^{1}$ Graduate School of Agriculture, Kyoto University \\ ${ }^{2}$ Research Fellow of Japan Society for the Promotion of Science \\ ${ }^{3}$ Graduate School of Natural Science and Technology, Shimane University \\ ${ }^{4}$ Fisheries Ecosystem Project Center, Shimane University \\ ${ }^{5}$ Faculty of Commerce, Doshisha University \\ *yoshih@life.shimane-u.ac.jp
}

Received: February 16, 2020; Accepted: August 24, 2020; Published: August 31, 2020

\begin{abstract}
We derive a Fokker Planck Equation (FPE) governing probability density functions (PDFs) of an impulsively controlled 1-D diffusion process in seasonal population management problems. Two interventions are considered: perfect (completely controllable) and imperfect interventions (not completely controllable). The FPE is an initial- and boundary-value problem subject to a non-local boundary condition along a moving boundary. We show that an finite volume method (FVM) with a domain transformation realizes a conservative discretization for the FPE. We demonstrate that the computed PDFs with the FVM and those with a Monte Carlo method agree well.
\end{abstract}

Keywords: Impulse control, Population management, Probability density function, Fokker Planck equation, Finite volume method, Domain transformation

\section{Introduction}

Controlling stochastic system dynamics through impulsive interventions can be effectively described in the framework of stochastic impulse control $[1,2,3,4]$. These problems often have threshold-type optimal intervention strategies such that the state variables are instantaneously moved from one threshold to another threshold, both of which are determined by the optimality principle [5]. Stochastic impulse control problems arise in a variety of research areas where decision-making plays a central role. Such examples include portfolio management $[6,7]$, central bank operation $[1,8,9]$, consumption and investment $[5,10]$, cash 


\section{Journal of Advanced Simulation in Science and Engineering}

management [11], and recently ecological management [12,13].

We can find the optimal policy in a stochastic impulse control problem by solving an optimality equation called Hamilton-Jacobi-Bellman Quasi-Variational Inequality (HJBQVI) [5]. Many studies have focused on the HJBQVIs and the associated optimal controls; however, less attention has been paid for the controlled dynamics despite its practical importance [12]. The optimally controlled stochastic dynamics can be statistically characterized by the probability density functions (PDFs). The Fokker-Planck Equation (FPE), or Kolmogorov's Forward Equation, is a degenerate parabolic conservative partial differential equation governing the PDF $[13,14]$. In this view, analyzing the controlled dynamics effectively reduces to solving the corresponding FPE. Only a few approaches have been made for PDFs of impulsively controlled stochastic dynamics, and they focus only on stationary problems $[13,15]$. This background motivates us to computing non-stationary PDEs of impulsively controlled stochastic dynamics because such problems naturally arise in seasonal animal population management problems [16]. We consider imperfectness of the intervention for a stationary problem as the previous study [15], with which we can establish a better real-based mathematical model. Hereafter, we call a model with perfect (imperfect) interventions simply as "perfect (imperfect) intervention" when there will be no confusion.

Motivated by the above-presented background, we derive and compute a new FPE that governs a non-stationary PDF of an impulsively controlled 1-D diffusion process arising in a seasonal fish-eating bird population management problem. We analyze a management problem of fish-eating bird population, the Great Cormorant, whose population changes seasonally. The bird population increases in spring and summer because there exists plenty of a kind of fish (Ayu) in rivers $[17,18]$. The FPE is subject to a non-local boundary condition along a moving boundary representing a seasonal killing threshold. We propose a domain transformation technique to rewrite the FPE in a moving domain to that in a fixed domain. A finite volume method (FVM) is then employed to discretize the FPE on the transformed domain, utilizing the exponentially fitting discretization $[13,15,19]$. The FVM is conservative and preserves non-negativity of numerical solutions, both of which are physical requirements to be equipped with computed PDFs. We demonstrate that the computed PDFs with the FVM and a Monte Carlo scheme agree well with each other. In addition, we find that the FPE approach is at most $O(\mathrm{~min})$, while Monte Carlo method at least $O($ day $)$ in our setting.

The rest of this paper is organized as follows. Section 2 introduces the model problem of non-stationary stochastic impulse control problems for both perfect and imperfect interventions. The FPE and its transformed counterpart are derived in Section 3. Section 4 presents and verifies the FVM for perfect and imperfect interventions through a comparison with a Monte Carlo method. Section 5 concludes this paper and proposes our research perspectives in future. 


\section{Stochastic impulse control model}

\subsection{Perfect intervention model}

A model stochastic impulse control problem is introduced following the previous studies $[12,15]$. We focus on a fish-eating bird population as an example, but the methodology presented in this paper can be applied to generic problems of single-species population management. The decision-maker, the manager of an animal population who is in charge of controling the population, can impulsively reduce the population through interventions (an example is gun-shooting). This assumption is justified if the interventions are taken in a much shorter timescale than that of the population dynamics.

The population size at the time $t$ is denoted as $X_{t} \geq 0$ which is right continuous with left limits as in the conventional impulse control models [5]. We consider a control problem under full information [5] in a finite horizon $[0, T]$. The temporal evolution of $X_{t}(t \geq 0)$ is governed by the Itô's type stochastic differential equation (SDE) subject to impulsive interventions

$$
\left\{\begin{array}{c}
\mathrm{d} X_{t}=X_{t}\left(\mu(t) \mathrm{d} t+\sigma(t) \mathrm{d} B_{t}\right), \tau_{i} \leq t<\tau_{i+1} \\
X_{\tau_{i}}=X_{\tau_{i-}}-\zeta_{i}
\end{array}\right.
$$

with the initial population $X_{0-}=x \geq 0$, where $\mu(t)>0$ is the time-dependent drift as the deterministic growth rate, $\sigma(t)>0$ with $\mu>\sigma^{2}$ is the time-dependent volatility modulating the stochastic disturbance involved in the population dynamics, $B_{t}(t \geq 0)$ is the 1-D standard Brownian motion defined on a usual complete probability space [20], $\tau_{i}$ ( $i=0,1,2, \cdots, N, \tau_{0}=0$ ) is the sequence of strictly increasing stopping times at which the intervention is taken, and $\zeta_{i}$ represents the killed population at the time $\tau_{i}$. Here, $X_{0-}=\lim _{s \rightarrow+0} X_{0-s}$ means the left limit of $X_{t}$ at $t=0$, and $N$ is the total number of the intervention during the period $[0, T]$. Notice that this is a stochastic variable as well. The model parameters in (1), assuming a stationary state, can be identified by field surveys [18]. In the perfect intervention model, we can reduce the number of the population exactly. For each intervention, the following linear cost is incurred:

$$
K\left(\zeta_{i}\right)=k_{1} \zeta_{i}+k_{0} \quad\left(\zeta_{i}>0\right)
$$

where $k_{1}>0$ is the proportional cost (costs for killing population) and $k_{0} \geq 0$ is the fixed cost (labor and implementation costs). In the context of the Great Cormorant management, the proportional cost corresponds to that of bullets, and the fixed costs to that of labor costs [21].

The following performance index $J$ represents the expected net profit of the decision-maker [12]:

$$
J(x, t ; \eta)=\mathrm{E}\left[\int_{t}^{T} e^{-\delta(s-t)}\left(u X_{s}^{M}-d X_{s}^{m}\right) \mathrm{d} s-\sum_{i, t \leq \tau_{i} \leq T} e^{-\delta\left(\tau_{i}-t\right)} K\left(\zeta_{i}\right) \mid X_{t-}=x\right],
$$

where $\delta \geq 0$ is the discount rate, and $u, d, M$ and $m$ are positive constants. The larger (smaller) $\delta$ means that the decision-maker is more (less) myopic. In the right-hand side of (3), the term $u X_{s}^{M}$ represents the ecological utility provided by the population, the term $d X_{s}^{m} \quad$ represents the disutility provided by the population, and the last summation term represents the (discounted) total cost for taking the intervention. As explained in [18], (3) is the 
simplest lumped representation of the sum of the utility and disutility. The sum $-u X_{s}^{M}+d X_{s}^{m}$ is convex and unimodal, and thus takes a global minimum with some $X_{s}>0$. We define the management policy $\eta$ as the couple $\eta=\left(\tau_{i}, \zeta_{i}\right)_{0 \leq i \leq N}$.

Following the standard methodology for impulse control problems of diffusion processes $[1,2]$, it turns out that the following time-dependent threshold-type policy with some threshold values $\bar{x}(t)$ and $\underline{x}(t) \quad(0<\underline{x}(t)<\bar{x}(t))$ is optimal [22]:

If $X_{t-}<\bar{x}(t)$ then no intervention is taken, and only if $X_{t-}=\bar{x}(t)$ then the intervention is immediately taken and $X_{t-}$ is impulsively reduced to $\underline{x}(t) \quad\left(X_{t}=\bar{x}(t)\right)$.

The above-mentioned policy means that $X_{t}(t \geq 0)$ is confined in $[0, \bar{x}(t)]$ if $X_{0-} \leq \bar{x}(0)$. A sample path of $X_{t}$ with the optimal management policy is plotted in Fig. 1. Hereafter, we assume that the threshold values $\bar{x}(t)$ and $\underline{x}(t)$ are already found based on some optimality principle because our focus is the stochastic dynamics rather than the optimal policy. These thresholds can be found by solving the HJBQVI $[1,2,3,22]$ associated with (1) and (3). Thus, we assume that the two thresholds $\bar{x}(t)$ and $\underline{x}(t)$ have already been given.

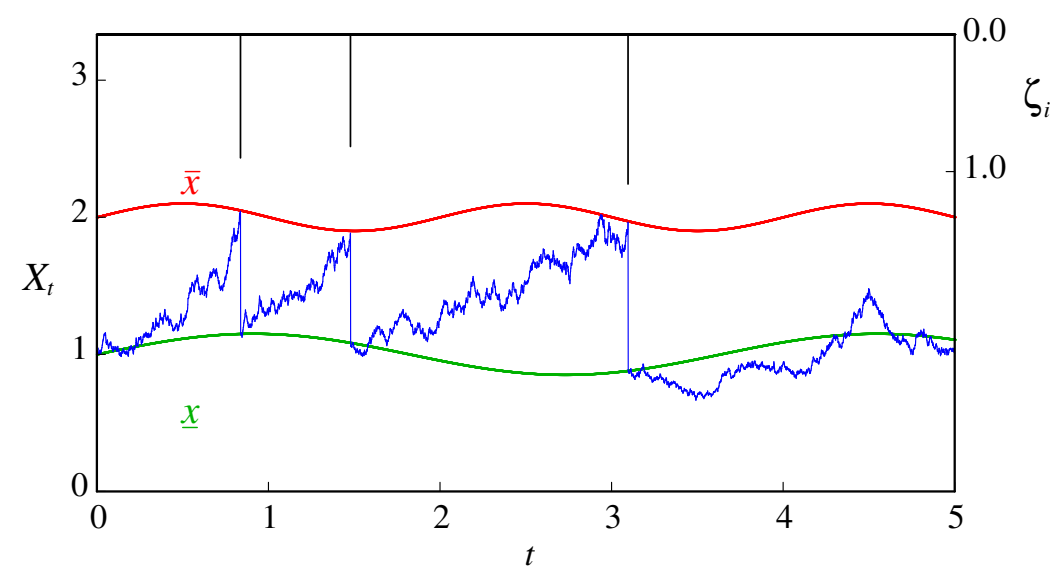

Figure 1: The sample path of $X_{t}$ (blue) with the thresholds $\bar{x}(t)$ (red), $\underline{x}(t)$ (green) and the magnitude of control $\zeta_{i}$ (black).

\subsection{Imperfect intervention model}

A drawback of the perfect intervention is that the human interventions are accurately achieved in the sense that paying the cost $K(\zeta)$ can reduce the population exactly by $\zeta$. However, it is more reasonable to assume that such an exact intervention is not realized in practice, but that there exists uncertainty in the realization [15]. This means that paying the cost $K(\zeta)$ leads to reduction of the population, but the effectively reduced population is not $\zeta$ but $Z \zeta$, where $Z>0$ is a stochastic variable that is not predictable for the decision-maker. In other words, the population truly reduced at $\tau_{i}$ is $(\bar{x}-\underline{x}) Z_{i}$ because of the 
imperfectness of interventions, which equals to the perfect model only if $Z_{i}=1$. In what follows, we call an optimal control with the uncertainty $Z_{i}$ as an imperfect intervention. Hereafter, for the sake of brevity of descriptions, we set the amount of reduced population at $\tau_{i}$ by the imperfect optimal control as $\bar{x}-Z_{i}$ with an abuse of notation; again it equals $\bar{x}-\underline{x}$ if $Z_{i}=\underline{x}$. We assume that each $Z_{i}(i=0,1,2, \ldots, N)$ is related to $\underline{x}$, and is i.i.d. Furthermore, each $Z_{i}$ is assumed to be generated by a PDF $q=q(z)$ supported on $[a(t), b(t)]$ such that $0<\bar{x}-Z_{i}<\bar{x}$ or equivalently $a(t)<\underline{x}(t)<b(t)$.

\section{Fokker Planck equation}

\subsection{Derivation of the FPE for perfect interventions}

Following the physical consideration that the population hitting the threshold $\bar{x}(t)$ is immediately reduced to $\underline{x}(t)[13,15]$, the FPE governing the PDF $p=p(x, t)$ associated with a time-dependent impulse control model for perfect interventions follows as

$$
\frac{\partial p}{\partial t}+\frac{\partial F}{\partial x}=0,0<x<\bar{x}(t), \quad x \neq \underline{x}(t), 0<t<T
$$

with the flux given by

$$
F=\mu x p-\frac{\partial}{\partial x}\left(\frac{\sigma^{2} x^{2}}{2} p\right)=\operatorname{rxp}-D x^{2} \frac{\partial p}{\partial x}
$$

and the non-local boundary condition based on the mass conservation

$$
F_{\underline{x}(t)-}+F_{\bar{x}(t)-}=F_{\underline{x}(t)+},
$$

and the standard boundary conditions $[13]$

$$
p(\bar{x}(t), t)=0
$$

and

$$
F_{0}=0 \text {. }
$$

Here, $r=\mu-\sigma^{2}>0$ and $D=0.5 \sigma^{2}$. The PDF should satisfy the conservation condition

$$
\int_{0}^{\bar{x}(t)} p(x, t) \mathrm{d} x=1 \text {. }
$$

The ordinal boundary condition (7) means that the population hitting the upper threshold $\bar{x}(t)$ is immediately transported into the lower threshold $\underline{x}(t)$. Notice that Yaegashi et al. [13] discussed only the problem with time-independent thresholds.

Partially differentiating equation (9) with respect to $t$ and applying the Leibnitz's rule [19] with the help of (7)-(9) yields

$$
\begin{aligned}
\frac{\mathrm{d}}{\mathrm{d} t} \int_{0}^{\bar{x}(t)} p(x, t) \mathrm{d} x & =\int_{0}^{\bar{x}(t)} \frac{\partial p}{\partial t} \mathrm{~d} x+p(\bar{x}(t), t) \bar{x}^{\prime}-p(0, t) \frac{\mathrm{d}}{\mathrm{d} t}(0) \\
& =\int_{0}^{\bar{x}(t)}-\frac{\partial F}{\partial x} \mathrm{~d} x+p(\bar{x}(t), t) \bar{x}^{\prime} \\
& =-F_{\bar{x}(t)}+F_{0}+p(\bar{x}(t), t) \bar{x}^{\prime} \\
& =0
\end{aligned},
$$

meaning that the following condition should be imposed to guarantee the normalization (9):

$$
F_{\bar{x}(t)}=p(\bar{x}(t), t) \bar{x}^{\prime}=0 .
$$


With this condition, it is straightforward to see that the FPE (9) is indeed conservative.

\subsection{Derivation of the transformed FPE for perfect interventions}

The FPE (4) is defined in a moving domain. We rewrite the equation to a problem in a fixed domain using a simple domain transformation technique, with which an efficient FVM for its numerical resolution can be derived. We consider the transformation inspired from [23]:

$$
(0, \bar{x}) \mapsto(0,1), \text { or equivalently }\left\{\begin{array}{l}
s=t \\
y=\bar{x}^{-1} x
\end{array}\right.
$$

With an abuse of notation, we still denote the PDF with the transformed variable as $p=p(y, s)$. By (12), the partial derivatives with respect to time and space is calculated as

$$
\begin{aligned}
\frac{\partial p}{\partial t} & =\frac{\partial p}{\partial s} \frac{\partial s}{\partial t}+\frac{\partial p}{\partial y} \frac{\partial y}{\partial t} \\
& =\frac{\partial p}{\partial s}+\frac{\partial p}{\partial y} \frac{\partial}{\partial t}\left(\bar{x}^{-1} x\right) \\
& =\frac{\partial p}{\partial s}-y \frac{\bar{x}^{\prime}}{\bar{x}} \frac{\partial p}{\partial y}
\end{aligned}
$$

and

$$
\begin{aligned}
\frac{\partial p}{\partial x} & =\frac{\partial p}{\partial s} \frac{\partial s}{\partial x}+\frac{\partial p}{\partial y} \frac{\partial y}{\partial x} \\
& =\frac{\partial p}{\partial y} \frac{\partial}{\partial x}\left(\bar{x}^{-1} x\right) . \\
& =\frac{1}{\bar{x}} \frac{\partial p}{\partial y}
\end{aligned}
$$

Substituting (13) and (14) into the FPE (4) yields

$$
\begin{aligned}
\frac{\partial p}{\partial t}+\frac{\partial F}{\partial x} & =\left(\frac{\partial p}{\partial s}-y \frac{\bar{x}^{\prime}}{\bar{x}} \frac{\partial p}{\partial y}\right)+\frac{1}{\bar{x}} \frac{\partial F}{\partial y} \\
& =\left(\frac{\partial p}{\partial s}-y \frac{\bar{x}^{\prime}}{\bar{x}} \frac{\partial p}{\partial y}\right)+\frac{\partial}{\partial y}\left(r y p-D y^{2} \frac{\partial p}{\partial y}\right) \text { in }(y, s) \in(0,1) \times(0, T) . \\
& =0
\end{aligned}
$$

In addition, the transformed FPE (15) can be written in a conservative form as

$$
\begin{aligned}
& \bar{x} \frac{\partial p}{\partial s}-y \bar{x}^{\prime} \frac{\partial p}{\partial y}+\bar{x} \frac{\partial}{\partial y}\left(r y p-D y^{2} \frac{\partial p}{\partial y}\right) \\
= & \frac{\partial(\bar{x} p)}{\partial s}-\frac{\partial}{\partial y}\left(\bar{x}^{\prime} y p\right)+\frac{\partial}{\partial y}\left(\bar{x} r y p-D \bar{x} y^{2} \frac{\partial p}{\partial y}\right) \\
= & \frac{\partial(\bar{x} p)}{\partial s}+\frac{\partial}{\partial y}\left(\left(\bar{x} r-\bar{x}^{\prime}\right) y p-D \bar{x} y^{2} \frac{\partial p}{\partial y}\right) \quad \text { in }(y, s) \in(0,1) \times(0, T) \\
= & \frac{\partial(\bar{x} p)}{\partial s}+\frac{\partial \bar{F}}{\partial y} \\
= & 0
\end{aligned}
$$

with 


$$
\bar{F}=\left(\bar{x} r-\bar{x}^{\prime}\right) y p-D \bar{x} y^{2} \frac{\partial p}{\partial y}=-\frac{\bar{x}^{\prime} x}{\bar{x}} p(x, t)+F .
$$

The boundary conditions (7) and (8) are transformed as

$$
p(1, s)=p(\bar{x}(t), t)=0
$$

and

$$
\bar{F}_{0}=-\frac{\bar{x}^{\prime} \cdot 0}{\bar{x}} p(0, t)+F_{0}=0 .
$$

The inner boundary condition (6) is transformed as

$$
\left[-\bar{x}^{\prime} \underline{y} p(\underline{y}-, s)+\bar{F}_{\underline{y}(s)-}\right]+\left[-\bar{x}^{\prime} \cdot 1 \cdot p(1-, s)+\bar{F}_{1-}\right]=-\bar{x}^{\prime} \underline{y p}(\underline{y}+, s)+\bar{F}_{\underline{y}(s)+},
$$

where $\underline{y}(s)$ represents transformed lower threshold. We assume continuity of the PDF $p(y, s)$ for $0<s$ and $0<y<1$. Then, (6) can be rewritten as

$$
\bar{F}_{\underline{y}(s)-}+\bar{F}_{1-}=\bar{F}_{\underline{y}(s)+} .
$$

The transformed FPE (15) is still conservative because of

$$
\begin{aligned}
\frac{\mathrm{d}}{\mathrm{d} t} \int_{0}^{\bar{x}(t)} p(x, t) \mathrm{d} x & =\frac{\mathrm{d}}{\mathrm{d} t} \int_{0}^{1} p(y, s) \bar{x} \mathrm{~d} y \\
& =\int_{0}^{1} \frac{\partial \bar{F}}{\partial y} \mathrm{~d} y \\
& =-\frac{\bar{x}^{\prime} \bar{x}}{\bar{x}} p(\bar{x}(t), t)+F_{\bar{x}(t)}-0 . \\
& =-p(\bar{x}(t), t) \bar{x}^{\prime}+p(\bar{x}(t), t) \bar{x}^{\prime} \\
& =0
\end{aligned}
$$

\subsection{Derivation of the transformed FPE for imperfect interventions}

For the imperfect intervention cases, from a physical consideration, the right flux $F_{\bar{x}(t)-}$ is distributed in $[a(t), b(t)]$ according to the PDF $q(x)$ such that $\int_{a(t)}^{b(t)} q(x) \mathrm{d} x=1$, which represents the imperfectness of interventions. At $x$ such that $a(t) \leq x \leq b(t)$, the point-wise distributed flux is $q(x) F_{\bar{x}(t)-}$. Note that the right flux $F_{\bar{x}(t)-}$ is equal to the distributed flux to the domain. Therefore, we should have

$$
\int_{a(t)}^{b(t)} q(x) F_{\bar{x}(t)-} \mathrm{d} x=F_{\bar{x}(t)-} .
$$

Considering the above-mentioned argument, the FPE for imperfect cases is derived as

$$
\frac{\partial p}{\partial t}+\frac{\partial F}{\partial x}=\chi_{[a(t), b(t)]} q(x) F_{\bar{x}(t)-}, 0<x<\bar{x}(t), 0<t<T
$$

with the flux (5) and the boundary conditions (7) and (8). Here, $\chi_{[a(t), b(t)]}$ is the indicator function such that $\chi_{[a(t), b(t)]}=1$ when $x \in[a(t), b(t)]$ and $\chi_{[a(t), b(t)]}=0$ otherwise. Notice that this FPE is a time-dependent counterpart of that in [15]. The PDF $p$ in this case also satisfies the condition (9). Indeed, we have 


$$
\begin{aligned}
\frac{\mathrm{d}}{\mathrm{d} t} \int_{0}^{\bar{x}(t)} p(x, t) \mathrm{d} x & =\int_{0}^{\bar{x}(t)} \frac{\partial p}{\partial t} \mathrm{~d} x+p(\bar{x}(t), t) \bar{x}^{\prime}-p(0, t) \frac{\mathrm{d}}{\mathrm{d} t}(0) \\
& =\int_{0}^{\bar{x}(t)}\left[-\frac{\partial F}{\partial x}+\chi_{[a(t), b(t)]} q(x) F_{\bar{x}(t)-}\right] \mathrm{d} x+p(\bar{x}(t), t) \bar{x}^{\prime} . \\
& =-F_{\bar{x}(t)}+F_{0}+F_{\bar{x}(t)-}+p(\bar{x}(t), t) \bar{x}^{\prime} \\
& =0
\end{aligned}
$$

As for the perfect interventions, we transform (24) using the transformation (12) as

$$
\frac{\partial(\bar{x} p)}{\partial s}+\frac{\partial \bar{F}}{\partial y}=\chi_{[\bar{a}(s), \bar{b}(s)]} q(y) \bar{F}_{1-}
$$

where $\bar{a}(s)$ and $\bar{b}(s)$ are the transformed $a(t)$ and $b(t)$, respectively. Note that the FPE for imperfect interventions reduces to that for perfect interventions if $q(x)=\delta(\underline{x})$, where $\delta(x)$ is the Dirac's delta concentrated at $x$.

\section{Finite volume method}

\subsection{FVM for perfect interventions}

Recall that there are two types of boundaries: the inner boundary $x=\underline{x}$ and the ordinary boundaries $x=0$ and $x=\bar{x}$. In the transformed spatial domain, they are $y=\underline{y}, y=0$, and $y=\bar{y}=1$, respectively. The domain $[0,1]$ is divided into $N+1$ cells and $N+1$ nodes $y_{i}$ as $0=y_{0}<y_{1}<\ldots<y_{M-1}<y_{M}<y_{M+1}<\ldots<y_{N-1}<y_{N}=1$ so that $y_{M}$ is the nearest node from $\underline{y}$ (Fig. 2). The nodes are located at the center of cells except at the ordinary boundary based on the cell-vertex framework [24]. The $i$ th cell except for ordinal boundary is composed of the domain $\left[y_{i-1 / 2}, y_{i+1 / 2}\right]$ where $y_{i-1 / 2}=\left(y_{i-1}+y_{i}\right) / 2$. The 0 th cell is the interval $\left[y_{0}, y_{1 / 2}\right]$ and the $N$ th cell is the interval $\left[y_{N-1 / 2}, y_{N}\right]$. The cells located at the ordinary boundaries ( $y=0$ and $y=\underline{y}$ ) are the half size of the other cells and the outside nodes are located at the points where ordinary boundary is located (not the center of cells). For the sake of brevity, we employ a uniform mesh; the length between each successive node is $\Delta y=1 / N$. The time increment for temporal discretization is denoted as $\Delta s$. The PDF $p$ approximated at the node $i$ and the time step $n$ is denoted as $p_{i}^{n}$. By integrating the transformed FPE (16) in the control volume $\left(y_{i-1 / 2}, y_{i+1 / 2}\right) \times\left(s_{n}, s_{n+1}\right)$, we obtain

leading to the FVM

$$
\begin{aligned}
\int_{s_{n}}^{s_{n+1}} \int_{y_{i-1 / 2}}^{y_{i+1 / 2}}\left[\frac{\partial(\bar{x} p)}{\partial s}+\frac{\partial \bar{F}}{\partial y}\right] \mathrm{d} y \mathrm{~d} s & =\int_{s_{n}}^{s_{n+1}} \int_{y_{i-1 / 2}}^{y_{i+1 / 2}} \frac{\partial(\bar{x} p)}{\partial s} \mathrm{~d} y \mathrm{~d} s+\int_{s_{n}}^{s_{n+1}} \int_{y_{i-1 / 2}}^{y_{i+1 / 2}} \frac{\partial \bar{F}}{\partial y} \mathrm{~d} y \mathrm{~d} s \\
& =\int_{s_{n}}^{s_{n+1}} \frac{\partial(\bar{x} p)}{\partial s} \Delta y \mathrm{~d} s+\int_{s_{n}}^{s_{n+1}}[\bar{F}]_{y_{i-1 / 2}}^{y_{i+1 / 2}} \mathrm{~d} s \\
& =[\bar{x} p]_{s_{n}}^{s_{n+1}} \Delta y+[\bar{F}]_{y_{i-1 / 2}}^{y_{i+1 / 2}} \Delta s \\
& =\left(\bar{x}^{n+1} p_{i}^{n+1}-\bar{x}^{n} p_{i}^{n}\right) \Delta y+\left(\bar{F}_{i+1 / 2}^{n}-\bar{F}_{i-1 / 2}^{n}\right) \Delta s \\
& =0
\end{aligned}
$$




$$
p_{i}^{n+1}=\frac{1}{\bar{x}^{n+1}}\left[\bar{x}^{n} p_{i}^{n}-\left(\bar{F}_{i+1 / 2}^{n}-\bar{F}_{i-1 / 2}^{n}\right) \frac{\Delta s}{\Delta y}\right],
$$

where $\bar{F}_{i+1 / 2}^{n}$ is the numerical flux $[13,19]$. By (17), the numerical flux is set as

$$
\bar{F}_{i+1 / 2}^{n}=\frac{-V_{i+1 / 2}^{n} e^{\mathrm{Pe}^{n}}}{1-e^{\mathrm{Pe}^{n}}} p_{i}^{n}+\frac{V_{i+1 / 2}^{n}}{1-e^{\mathrm{Pe}^{n}}} p_{i+1}^{n}=\alpha_{i}^{n} p_{i}^{n}+\beta_{i}^{n} p_{i+1}^{n}
$$

with

$$
\mathrm{Pe}^{n}=\frac{V_{1+1 / 2}^{n} \Delta y}{\varepsilon_{i+1 / 2}^{n}}, \quad V_{i+1 / 2}^{n}=\left[\left(\mu-\sigma^{2}\right) \bar{x}^{n}-\bar{x}^{\prime n}\right]\left(\frac{y_{i}+y_{i+1}}{2}\right), \quad \varepsilon_{i+1 / 2}^{n}=\frac{\sigma^{2}}{2}\left(\frac{y_{i}+y_{i+1}}{2}\right)^{2} \bar{x}^{n} .
$$

For the boundary conditions, the Dirichlet condition (18) is directly specified at $y=1$, the flux condition (19) is imposed in the formula (28) at the cell 0, which has the cell size $\Delta y / 2$, and the inner boundary condition (21) is handled as

$$
p_{M}^{n+1}=\frac{1}{\bar{x}^{n+1}}\left[\bar{x}^{n} p_{M}^{n}-\left(\bar{F}_{M+1 / 2}^{n}-\bar{F}_{M-1 / 2}^{n}-\bar{F}_{N-1 / 2}^{n}\right) \frac{\Delta s}{\Delta y}\right],
$$

where $\bar{F}_{N-1 / 2}^{n}$ is the flux at the surface between the nodes $N-1$ and $N$.

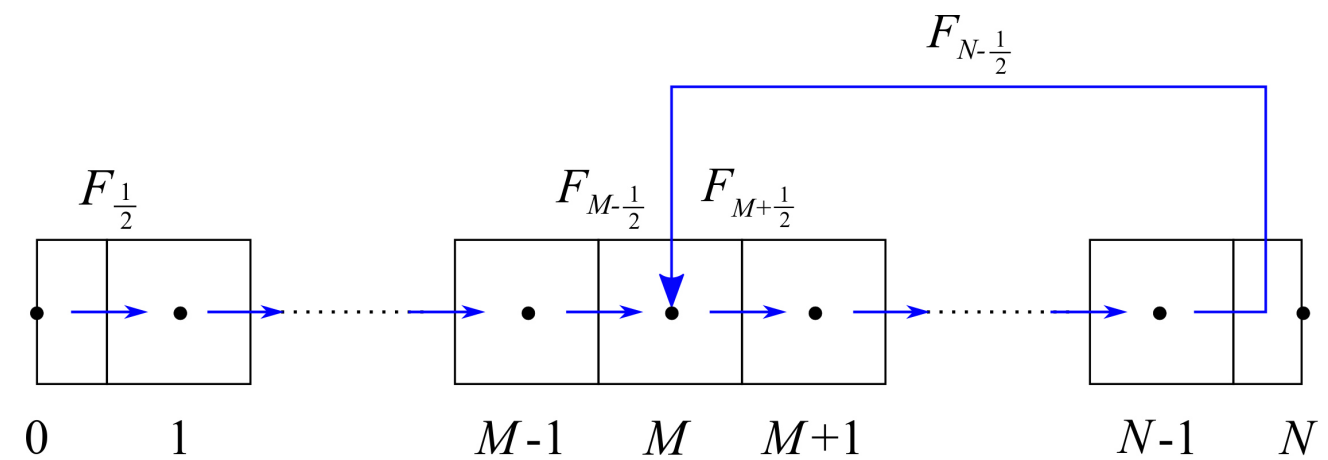

Figure 2: Schematic diagrams of nodes, cells $(i=0, \cdots, M, \cdots, N)$ and fluxes $F_{i+1 / 2}$. The flux $F_{N-1 / 2}$ flows into the cell $M$ irreversibly.

We prove that the presented FVM is conservative and non-negative for sufficiently small $\Delta s$ following the similar procedure in Yaegashi et al. [13]. We also confirm the mass conservation property in the latter parts.

The computed PDF of the FPE for perfect interventions by the FVM is validated with a computational result of a standard Monte Carlo method with the Mersenne twister [25] and Ziggurat algorithm [26]. The SDE (1) is directly discretized with the conventional Euler-Maruyama method [27]. The computed PDF and the histogram generated with the Monte Carlo method are compared for the parameter values $\mu=0.17, \sigma=0.2$, $\bar{x}(t)=2+A \sin (2 \pi t), \underline{x}(t)=1+A \cos (2 \pi t)$ with $A=0.5$, and $X_{0-}=1.5$. We assume that both thresholds $\bar{x}(t)$ and $\underline{x}(t)$ periodically change as the time elapses due to seasonal change in the population and the environment. Note that the parameters $\mu$ and $\sigma$ should be time-dependent for more realistic numerical simulation; however, they are assumed to be time-independent in this section because our focus in this paper is physically validating the FPE discretized with the FVM. Notice that stability and accuracy of the FVM for stationary problems where the thresholds are time-independent have already been verified in [15] through a comparison between numerical and exact solutions. The convergence speed of the FVM in the stationary case was found to be second-order. 


\section{Journal of Advanced Simulation in Science and Engineering}

We set the time increment $\Delta s=10^{-4}$ and the number of the cells $N=200$ in the FVM both for perfect and imperfect cases. This temporal resolution has been preliminary checked to be sufficiently small for stable numerical computation; the numerical solution does not change with a smaller time increment. For the Monte Carlo method, the time increment is set as $\Delta t=5.0 \times 10^{-4}$. The total number of generated sample paths is $5.0 \times 10^{6}$ and the interval for the histogram is set as $10^{-3}$. We compare the results by the FPE and by the Monte Carlo method at two different times, $t=1.1$ and $t=2.1$. Fig. 3 and Fig. 4 compare the results for perfect interventions by the FPE and the Monte Carlo method at $t=1.1$ and $t=2.1$, respectively. At $t=1.1$, the first and the second moments are 0.797 and 0.728 with the FPE, while those by the Monte Carlo method are 0.802 and 0.736 , respectively. At $t=2.1$, the first and the second moments are 0.820 and 0.741 with the FPE, while those by the Monte Carlo method are 0.824 and 0.746 , respectively. These results demonstrate good agreement between two different approaches. The computed results graphically show that the numerical solution with the FVM correctly captures the unimodal (Fig. 4) and bimodal nature (Fig. 3) of the numerical solution with the Monte Carlo method. We emphasize here that the Monte Carlo method and FPE are based on the theoretically different principle; the Monte Carlo method solves the dynamics (SDE) directly, while the FPE considers the balance and the flow of PDFs. An important result is that these fundamentally different methods computed the very similar PDFs.

For considering the computational time, a histogram for perfect interventions computed by the FVM and by the Monte Carlo method at $t=1.1$ with the number of the cells $N=400 \quad$ (Fig. 5) and a histogram for perfect interventions computed by the FVM and by the Monte Carlo method at $t=1.1$ with the time increment $\Delta s=5.0 \times 10^{-5}$ (Fig. 6) are provided. The black curve is the result with $N=200$ and $\Delta s=1.0 \times 10^{-4}$ (the same with Fig. 3) and red curve is the result with $N=400$ or $\Delta s=5.0 \times 10^{-5}$ (finer grid). Smaller time increment does not change the PDF and using a twice finer mesh enables us to capture the peak of the PDF more accurately. The smaller peak with FVM in Fig. 3 is therefore considered to be caused by the numerical diffusion associated with the exponentially-fitting discretization. Noting that the result with $N=400$ and $\Delta s=5.0 \times 10^{-5}$ is the same with the result with $N=400$ and $\Delta s=1.0 \times 10^{-4}$. The computational time is totally different. The computational times are still at most $O(\min )$ for FVM: for Monte Carlo method at least $O($ day) in our setting. Numerically solving the FPE is time saving and has satisfactory accuracy. A contour map of $p(x, t)$ for perfect interventions from $t=0$ to $t=T$ where $T=3$ is calculated by the FVM and shown in Fig. 7.

In addition, we investigate conservative property of the FVM because the original FPE (4) and the transformed one (16) are both conservative equations; the mass should be conserved. Table 1 shows mass errors at each time, which is defined as

$$
\text { Mass error }=\left|1-\int_{0}^{\bar{x}(t)} p(x, t) \mathrm{d} x\right| .
$$

According to Table 1, mass errors at each time is less than $10^{-13}$, which is sufficientlly small. Moreover, the mass error does not increase as the time evolves. 


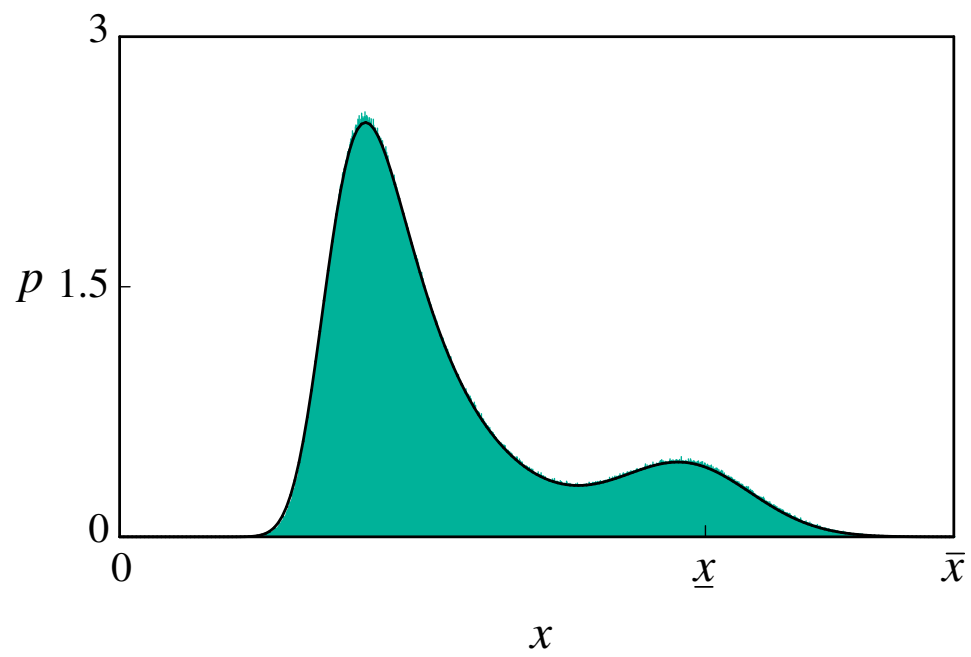

Figure 3: Histogram for perfect interventions computed by the FVM (black) and by the Monte Carlo method (green) at $t=1.1$.

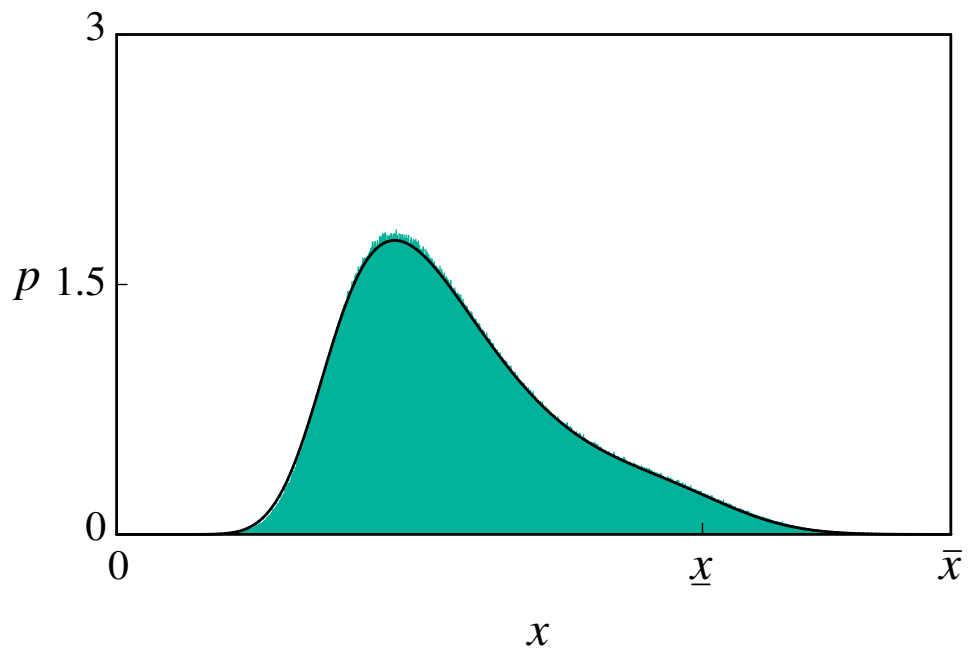

Figure 4: Histogram for perfect interventions computed by the FVM (black) and by the Monte Carlo method (green) at $t=2.1$. 


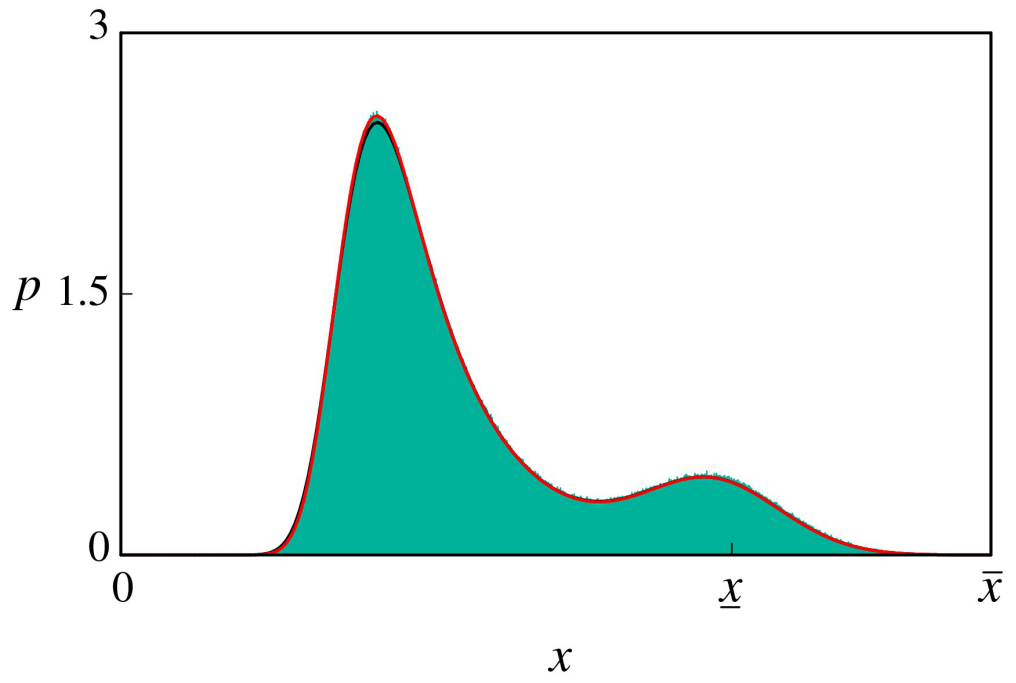

Figure 5: Histogram for perfect interventions computed by the FVM (black curve is Fig. 3 and red curve is finer) and by the Monte Carlo method at $t=1.1$ with the number of the cells $N=400$



Figure 6: Histogram for perfect interventions computed by the FVM (black curve is Fig. 3 and red curve is finer) and by the Monte Carlo method at $t=1.1$ with the time increment $\Delta s=5.0 \times 10^{-5}$. 


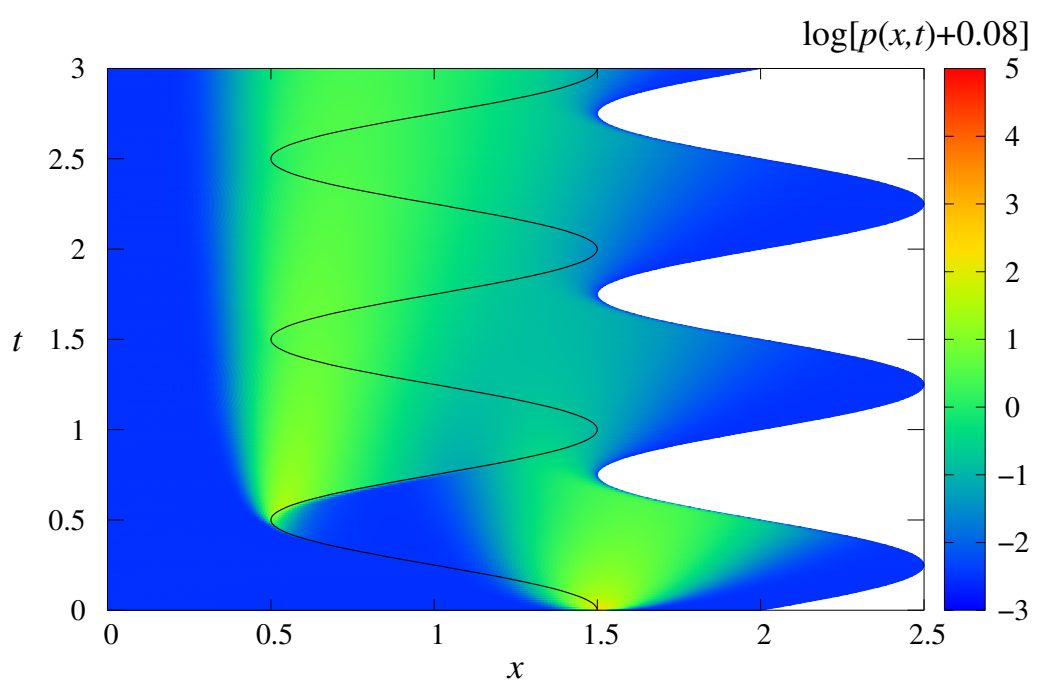

Figure 7: Contour map of $p(x, t)$ for perfect interventions from $t=0$ to $t=T$ with $\underline{x}(t)$ (black curve) calculated by the FVM in perfect intervention case.

Table 1: Time evolution of the mass error for the perfect FPE

\begin{tabular}{lllllll}
\hline Time & $t=5$ & $t=10$ & $t=15$ & $t=20$ & $t=25$ & $t=30$ \\
\hline Mass error & $7.55 \times 10^{-15}$ & $4.06 \times 10^{-14}$ & $8.37 \times 10^{-14}$ & $3.20 \times 10^{-14}$ & $3.66 \times 10^{-14}$ & $8.79 \times 10^{-14}$
\end{tabular}

\subsection{FVM for imperfect interventions}

We establish an FVM for imperfect interventions and validate it by comparing with the Monte Carlo method. For the sake of tractability, we consider the uniform distribution

$$
q(x)=\frac{1}{b(t)-a(t)}(a(t) \leq x \leq b(t)) .
$$

The other distributions, like the truncated Gaussian distributions, can be implemented by a slight modification of the algorithm. The spatial domain [0,1] is divided into $N+1$ cells and $N+1$ nodes $y_{i}$ as $0=y_{0}<y_{1}<\ldots<y_{L}<\ldots<y_{R}<\ldots<y_{N-1}<y_{N}=1$ so that $y_{\mathrm{L}}$ and $y_{\mathrm{R}}$ are the nearest nodes from $\bar{a}(s)$ and $\bar{b}(s)$. For discretization of the source term in the right hand side of the FPE for imperfect cases (24), we employ the naïve discretization [15]:

with

$$
p_{i}^{n+1}=\frac{1}{\bar{x}^{n+1}}\left[\bar{x}^{n} p_{i}^{n}-\left(\bar{F}_{i+1 / 2}^{n}-\bar{F}_{i-1 / 2}^{n}\right) \frac{\Delta s}{\Delta y}+S_{i}^{n} \Delta s\right]
$$

$$
S_{i}^{n}=\left\{\begin{array}{lc}
q_{i} \bar{F}_{1-} & y_{\mathrm{L}}<y_{i}<y_{\mathrm{R}} \\
0.5 q_{i} \bar{F}_{1-}^{n} & y_{i}=y_{\mathrm{L}}, y_{\mathrm{R}} \\
0 & \text { otherwise }
\end{array} .\right.
$$

We set $a(t)=0.9 \underline{x}(t)$ and $b(t)=1.1 \underline{x}(t)$. Note that the FVM is conservative and non-negative for sufficiently small $\Delta s$ following the similar procedure in [13]. 


\section{Journal of Advanced Simulation in Science and Engineering}

The computed PDF of the FPE for imperfect interventions by the FVM is compared with a numerical result of the Monte Carlo method. The computational condition is the same with the FVM for the perfect intervention. We compare the results by the FPE and by the Monte Carlo method at $t=1.1$. Fig. 8 compares the results by the FPE and by the Monte Carlo method at $t=1.1$. At $t=1.1$, the first and the second moments are 0.797 and 0.730 with the FPE, while those by the Monte Carlo method are 0.802 and 0.737 , respectively. The result demonstrates good agreement between two different approaches, verifying the validness of the FPE and the FVM. Again, the numerical solution with the FVM correctly captures qualitative changes of the shape of the PDF generated by the Monte Carlo method. A contour map of $p(x, t)$ for imperfect interventions from $t=0$ to $t=T$ where $T=3$ is calculated by the FVM and shown in Fig. 9. Comparing Fig. 7 and Fig. 9, the PDF for imperfect interventions is more dispersive than that for perfect interventions. In addition, mass error is also confirmed and is sufficiently small (Table 2).

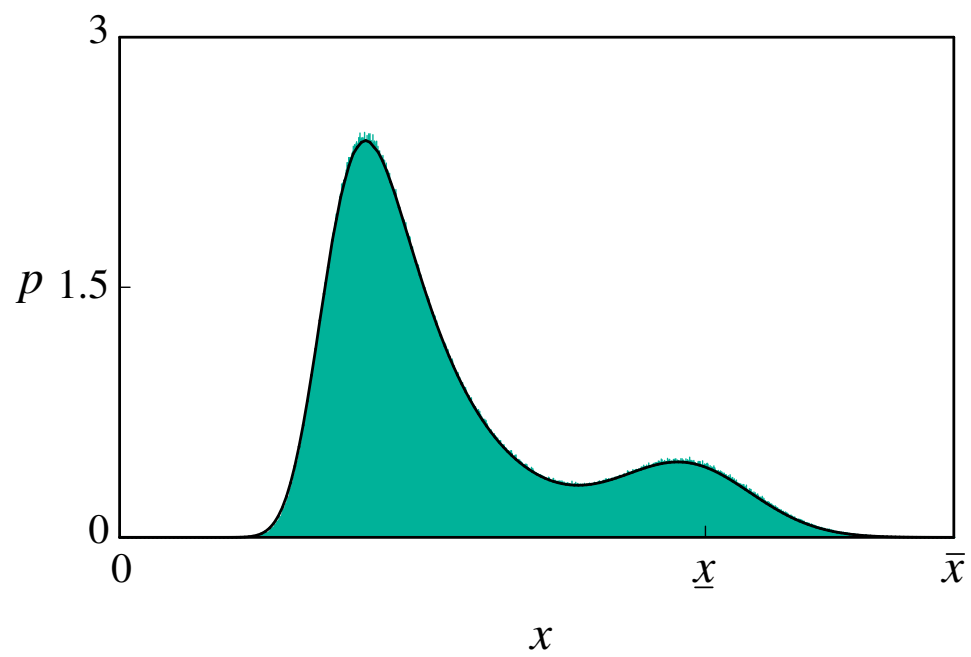

Figure 8: Histogram for imperfect interventions computed by the FVM (black) and by the Monte Carlo method (green) at $t=1.1$.

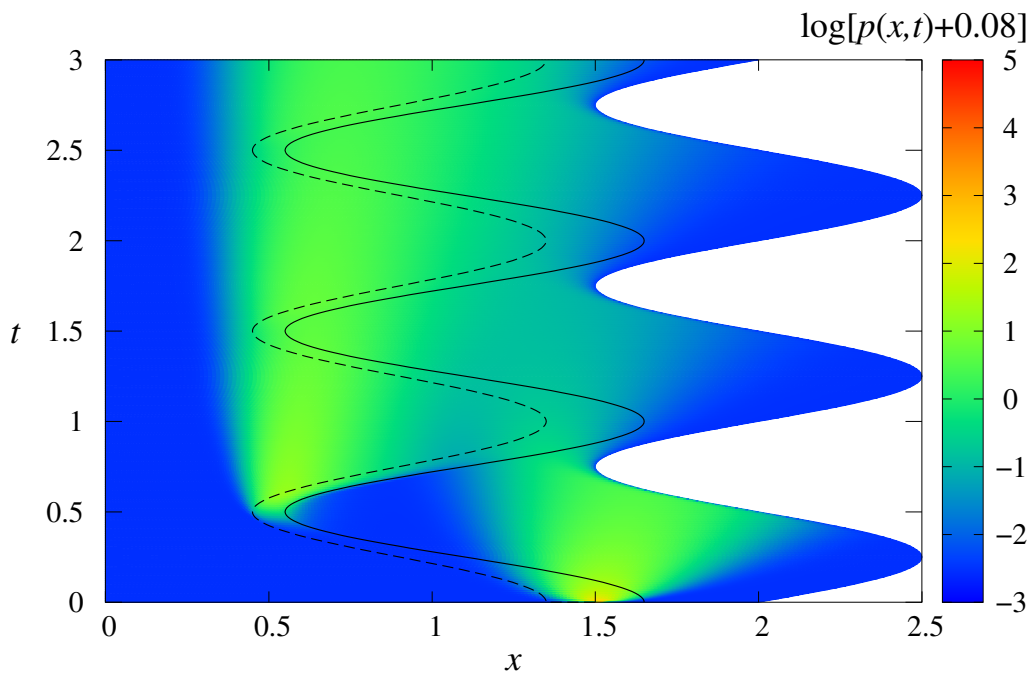




\section{Journal of Advanced Simulation in Science and Engineering}

Figure 9: Contour map of $p(x, t)$ for imperfect interventions from $t=0$ to $t=T$ calculated by the FVM. The dashed black curve is $a(t)$ and the black curve is $b(t)$.

Table 2: Time evolution of the mass error for the imperfect FPE

\begin{tabular}{lllllll}
\hline Time & $t=5$ & $t=10$ & $t=15$ & $t=20$ & $t=25$ & $t=30$ \\
\hline Mass error & $1.11 \times 10^{-15}$ & $2.11 \times 10^{-15}$ & $5.55 \times 10^{-16}$ & $4.11 \times 10^{-15}$ & $3.77 \times 10^{-15}$ & $4.44 \times 10^{-16}$ \\
& & & & & & \\
\hline
\end{tabular}

\section{Conclusions}

In this paper, we proposed the new FPEs of the non-stationary PDFs for a 1-D impulsively controlled diffusion process with perfect and imperfect interventions. We also proposed a domain transformation technique and the FVM for numerically solving the FPEs. The transformed FPEs and the FVM were numerically compared with a Monte Carlo method. The computational results demonstrated that the presented framework of the FVM can reproduce physically reasonable numerical solutions and more efficiently compute the PDF than the Monte Carlo method.

One of the remaining tasks is to handle PDFs in multi-dimensional problems in which other species dynamics are involved. Such a situation will be encountered in predator-prey systems. For example, we are interested in management of the Great Cormorant because they eat a fish with economic value, Ayu. The population of the fish changes as the season changes and then the bird population would accordingly change. This coupled problem is currently under investigation by the authors utilizing the collected data in an existing river.

\section{Acknowledgement}

JSPS Research Grant No. 17J09125, No. 19H03073 and No 18K01714 support this research. The authors thank Mr. Kohei Yokota for providing us a uniform random number generator based on Mersenne Twister.

\section{References}

[1] A. Cadenillas, F. Zapatero: Optimal central bank intervention in the foreign exchange market, Journal of Economic theory, 87:1 (1999), 218-242.

[2] M. Ohnishi, M. Tsujimura: An impulse control of a geometric Brownian motion with quadratic costs, European Journal of Operational Research, 168:2 (2006), 311-321.

[3] M. Tsujimura, A. Maeda: Stochastic control: Theory and applications, Asakura Shoten, 2016. (in Japanese)

[4] S. P. Van der Pijl, C. W. Oosterlee: An ENO-based method for second-order equations and application to the control of dike levels, Journal of Scientific Computing, 50:2 (2012), 462-492. 


\section{Journal of Advanced Simulation in Science and Engineering}

[5] B. Oksendal, A. Sulem: Optimal consumption and portfolio with both fixed and proportional transaction costs, SIAM Journal on Control and Optimization, 40:6 (2002), 1765-1790.

[6] S. Federico, P. Gassiat: Viscosity characterization of the value function of an investment-consumption problem in presence of an illiquid asset, Journal of Optimization Theory and Applications, 160:3 (2014), 966-991.

[7] R. Korn: Portfolio optimisation with strictly positive transaction costs and impulse control, Finance and Stochastics, 2:2 (1998), 85-114.

[8] G. Gagnon: Vanishing central bank intervention in stochastic impulse control, Annals of Finance, 15:1, (2019), 125-153.

[9] A. Bensoussan, H. Long, S. Perera, S. Sethi: Impulse control with random reaction periods: A central bank intervention problem, Operations Research Letters, 40:6 (2012), 425-430.

[10] S. Federico, M. Rosestolato, E. Tacconi: Irreversible investment with fixed adjustment costs: a stochastic impulse control approach, Mathematics and Financial Economics, 13:4 (2019), 579-616.

[11] S. Baccarin: Optimal impulse control for a multidimensional cash management system with generalized cost functions, European Journal of Operational Research, 196:1 (2009), 198-206.

[12] Y. Yaegashi, H. Yoshioka, K. Unami, M. Fujihara: A Stochastic Impulse Control Model for Population Management of Fish-Eating Bird Phalacrocorax Carbo and Its Numerical Computation, in Proc. 2018 Asia Simulation Conference, Kyoto, 2018, 425-438.

[13] Y. Yaegashi, H. Yoshioka, K. Tsugihashi, M. Fujihara: Analysis and computation of probability density functions for a 1-D impulsively controlled diffusion process, Comptes Rendus Mathematique, 357:3 (2019), 306-315.

[14] H. Risken: The Fokker-Planck Equation, Springer, Berlin, Heidelberg, 1996.

[15] H. Yoshioka, Y. Yaegashi, M. Tsujimura, M. Fujihara: Non-Local Fokker-Planck Equation of Imperfect Impulsive Interventions and its Effectively Super-Convergent Numerical Discretization, in Proc. 2019 Asia Simulation Conference, Singapore, 2019, 79-91.

[16] A. Jarnemo: Seasonal migration of male red deer (Cervus elaphus) in southern Sweden and consequences for management, European Journal of Wildlife Research, 54:2 (2008), 327-333.

[17] J. Tsuboi, A. Ashizawa:. Management of Great Cormorant colonies in Yamanashi Prefecture, Japanese Journal of Ornithology, 61:1, (2012), 38-45. 


\section{Journal of Advanced Simulation in Science and Engineering}

[18] Y. Yaegashi, H. Yoshioka, K. Unami, M. Fujihara: A singular stochastic control model for sustainable population management of the fish-eating waterfowl Phalacrocorax carbo, Journal of environmental management, 219 (2018), 18-27.

[19] H. Yoshioka, K. Unami: A cell-vertex finite volume scheme for solute transport equations in open channel networks, Probabilistic Engineering Mechanics, 31 (2013), 30-38.

[20] B. Oksendal: Stochastic differential equations: an introduction with applications, Springer Science \& Business Media, 2013.

[21] J. F. Glahn, M. E. Tobin, B. F. Blackwell: A science-based initiative to manage double-crested cormorant damage to southern aquaculture, USDA National Wildlife Research Center-Staff Publications, 532 (2000).

[22] Y. Yaegashi, H. Yoshioka, K. Tsugihashi, M. Fujihara: An exact viscosity solution to a Hamilton-Jacobi-Bellman quasi-variational inequality for animal population management, Journal of Mathematics in Industry, 9:5 (2019).

[23] M. Zhu, Y. Xu, J. Cao, The asymptotic profile of a dengue fever model on a periodically evolving domain, Applied Mathematics and Computation, 362 (2019), 124531.

[24] H. K. Versteeg, W. Malalasekera: An introduction to computational fluid dynamics: the finite volume method, Pearson education, 2007.

[25] M. Matsumoto, T. Nishimura: Mersenne twister: a 623-dimensionally equidistributed uniform pseudo-random number generator, ACM Transactions on Modeling and Computer Simulation (TOMACS), 8:1 (1998), 3-30.

[26] G. Marsaglia, W. W. TsangThe ziggurat method for generating random variables, Journal of statistical software, 5:8 (2000), 1-7.

[27] P. E. Kloeden, E. Platen, H. Schurz: Numerical solution of SDE through computer experiments, Springer Science \& Business Media, 2012. 